JOURNAL OF SYNCHROTRON RADIATION

ISSN 1600-5775

Received 1 September 2017

Accepted 9 November 2017

Edited by M. Yabashi, RIKEN SPring-8 Center, Japan

Keywords: microfocusing; X-ray optics; $\mathrm{KB}$ mirror; $\mathrm{X}$-ray free-electron laser; PAL-XFEL; NCI.

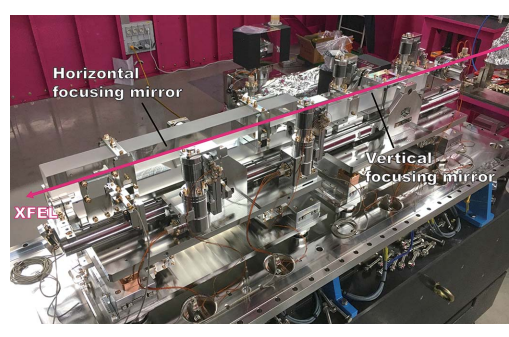

OPEN $\odot$ ACCESS

\section{Focusing X-ray free-electron laser pulses using Kirkpatrick-Baez mirrors at the NCI hutch of the PAL-XFEL}

\author{
Jangwoo Kim,* Hyo-Yun Kim, Jaehyun Park, Sangsoo Kim, Sunam Kim, \\ Seungyu Rah, Jun Lim and Ki Hyun Nam*
}

Pohang Accelerator Laboratory, POSTECH, Pohang, Gyeongbuk 37673, Republic of Korea.

*Correspondence e-mail: jkpal@postech.ac.kr, structure@postech.ac.kr

The Pohang Accelerator Laboratory X-ray Free-Electron Laser (PAL-XFEL) is a recently commissioned X-ray free-electron laser (XFEL) facility that provides intense ultrashort X-ray pulses based on the self-amplified spontaneous emission process. The nano-crystallography and coherent imaging (NCI) hutch with forward-scattering geometry is located at the hard X-ray beamline of the PAL-XFEL and provides opportunities to perform serial femtosecond crystallography and coherent X-ray diffraction imaging. To produce intense highdensity XFEL pulses at the interaction positions between the $\mathrm{X}$-rays and various samples, a microfocusing Kirkpatrick-Baez (KB) mirror system that includes an ultra-precision manipulator has been developed. In this paper, the design of a KB mirror system that focuses the hard XFEL beam onto a fixed sample point of the NCI hutch, which is positioned along the hard XFEL beamline, is described. The focusing system produces a two-dimensional focusing beam at approximately $2 \mu \mathrm{m}$ scale across the $2-11 \mathrm{keV}$ photon energy range. XFEL pulses of $9.7 \mathrm{keV}$ energy were successfully focused onto an area of size $1.94 \mu \mathrm{m} \times 2.08 \mu \mathrm{m}$ FWHM.

\section{Introduction}

In the X-ray regime, X-ray free-electron lasers (XFELs) produce unprecedented levels of brilliance, excellent spatial coherence and ultrafast pulse durations (McNeil \& Thompson, 2010). These unique properties provide opportunities to observe materials with high resolution, or facilitate radiation-damage-free and time-resolved studies using ultrashort pulse widths (Neutze et al., 2000; McNeil \& Thompson, 2010). In terms of XFEL facilities, the Linac Coherent Light Source (LCLS) (Emma et al., 2010) and SPring-8 Ångstrom Compact free-electron LAser (SACLA) (Ishikawa et al., 2012) facilities are both successfully operating hard XFELs. Recently, the Pohang Accelerator Laboratory (PAL-XFEL) (Ko et al., 2017) and the European XFEL (Decking \& Limberg, 2013) completed self-amplified spontaneous emission (SASE) commissioning and are now in operation. In addition, the SwissFEL (Milne et al., 2017) is now preparing for XFEL lasing.

Construction on the PAL-XFEL began in 2010 and was completed in 2015. The first phase of the PAL-XFEL includes two beamlines: a hard XFEL beamline and a soft XFEL beamline. The hard X-ray beamline consists of a $10 \mathrm{GeV}$ linear accelerator $(700 \mathrm{~m})$, undulator hall $(250 \mathrm{~m})$ and experimental hall $(60 \mathrm{~m})$ (Ko et al., 2017). The hard X-ray experimental hall consists of two experimental hutches (EHs). In the first $\mathrm{EH}$, which is called the X-ray scattering and 
spectroscopy (XSS) hutch, time-resolved experiments using the pump-probe technique are performed using primarily reflection geometry (Park, Eom et al., 2016). In the second $\mathrm{EH}$, which is called the nano-crystallography and coherent imaging (NCI) hutch, the most commonly performed studies are imaging studies that involve coherent X-ray diffraction imaging or serial femtosecond crystallography (Park, Kim et al., 2016). These experimental instruments incorporate forward-scattering geometries and support time-resolved experiments, which can be used separately or in combination. In such studies, it is essential to generate a high photon flux density at the sample position using X-ray focusing optics. Various optical focusing devices, such as Fresnel zone plates (David et al., 2011; Nilsson et al., 2012), refractive lenses (Schropp et al., 2013) and reflective mirrors (Yumoto et al., 2013; Mimura et al., 2014), are employed in other XFEL facilities. Among these, reflective mirrors achieve the highest focusing efficiencies over long working distances, and provide significant advantages in other applications. Thus, they were selected for use in our experimental devices. A microfocusing Kirkpatrick-Baez (KB) mirror system, which is a type of reflective mirror system, was developed for hard XFEL focusing applications at the PALXFEL. In this paper, we describe the main optical components for the hard XFEL beamline, the optical configuration of the microfocusing KB mirror system, and the current hard XFEL focusing capability at the PAL-XFEL.

\section{Optical configuration of the focusing mirrors}

The PAL-XFEL hard X-ray beamline consists of an undulator hall (UH), optical hutch $(\mathrm{OH})$ and two EHs. The microfocusing $\mathrm{KB}$ mirror system is located in the NCI hutch (Fig. 1). To transport the XFEL beam to the EHs, offset mirrors (OMs) or a double-crystal monochromator (DCM) in the $\mathrm{OH}$ are used for $\gamma$-ray shielding. The OMs and DCM are vertically offset by $30 \mathrm{~mm}$ and provide polychromatic or monochromatic X-rays, respectively. As a result, the XFEL beam from the UH enters the $\mathrm{OH}$ at a height of $1400 \mathrm{~mm}$ from the ground, and is delivered through the optical system at the $\mathrm{OH}$ to the EHs at $1430 \mathrm{~mm}$. The XFEL beam passing through either the OMs or DCM follows the same path and moves parallel to the EHs.

The focused beam size that can be obtained using the developed focusing mirror system is approximately $2 \mu \mathrm{m}$. The optical configuration was designed

Figure 1 and $5.365 \mathrm{~m}$, respectively. based on the expected location of the beam source and the theoretical source size. The optical design parameters are listed in Table 1. The radiation size and divergence of the hard XFEL source of PAL-XFEL were calculated using the GENESIS (Reiche, 1999) code simulation with various accelerator parameters (Parc et al., 2014). At a photon energy of $10 \mathrm{keV}$, the predicted source size and divergence are approximately $37.9 \mu \mathrm{m}$ (FWHM) and $1.7 \mu \mathrm{rad}$ (FWHM), respectively. The estimated incident beam size at the $\mathrm{KB}$ mirror is $280 \mu \mathrm{m}$. The length of the KB mirror is $600 \mathrm{~mm}$, the mirror employs a quartz substrate, the grazing incidence angle of the mirror is $2.6 \mathrm{mrad}$, and the focusing mirrors have a large spatial acceptance of $1.53 \mathrm{~mm}$ vertically and $1.51 \mathrm{~mm}$ horizontally, which is large enough to reflect spatially full XFEL pulses. The distance from the expected XFEL source position in the undulator to the centers of the vertical and horizontal focusing mirrors (VFM and HFM, respectively) are $141.685 \mathrm{~m}$ and $141.315 \mathrm{~m}$, respectively. The focal lengths of the VFM and HFM are $5.995 \mathrm{~m}$ and $5.365 \mathrm{~m}$, respectively. The ideal focused beam sizes are approximately $1.6 \mu \mathrm{m}$ (FWHM) and $1.4 \mu \mathrm{m}$ (FWHM) at $10 \mathrm{keV}$ in the vertical and horizontal directions, respectively, which are calculated by considering both the source size and the optical geometry. The long working

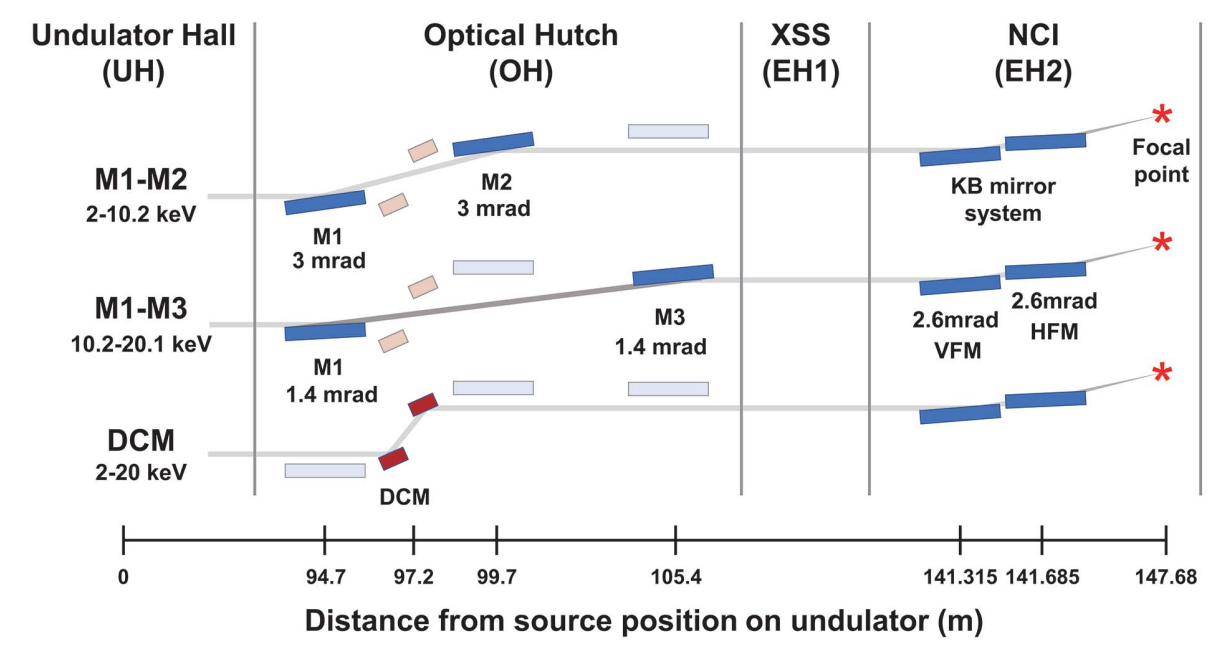

Schematic of the optical system of the NCI hutch at the hard X-ray beamline of the PAL-XFEL. The hard X-ray beamline includes three OMs (M1, M2 and M3) and a DCM at the OH. The KB mirror focusing system employs vertical and horizontal mirrors with focusing distances of $5.995 \mathrm{~m}$ 
distance of $5.065 \mathrm{~m}$ between the edge of the downstream mirror and the focal point is useful for combining various types of instruments with the focusing mirror system.

The effective area of the focusing mirrors is sagittally divided into two $10 \mathrm{~mm}$-wide optical strip lines. For high reflectivity, one of the effective areas was coated with a $25 \mathrm{~nm}$ thick carbon material, which has a theoretical reflectivity of greater than $99 \%$ at $2-11 \mathrm{keV}$. Another effective area was a bare quartz surface to minimize or eliminate the speckle pattern from the coating material. The carbon-coated area is intended for experiments requiring high photon flux, while the non-coated area is used for coherent diffraction imaging experiments requiring speckle-free XFEL pulses.

\section{Evaluation of focusing performance}

To verify the theoretically calculated focused beam size, we directed X-rays through the developed focusing mirror and mirror manipulation system at the NCI hutch. The XFEL pulses used in this study were generated by a polychromatic $\mathrm{X}$-ray passing through the OMs (i.e. a combination of M1 and M2) at the $\mathrm{OH}$. The photon energy was $9.7 \mathrm{keV}$ and the repetition rate was $10 \mathrm{~Hz}$. The complete beam path and $\mathrm{KB}$ mirrors were operated in a vacuum of about $10^{-8}$ torr. Fourway slits were placed upstream and downstream of the KB mirrors to remove the halo and suppress parasitic scattering from the edge of the mirror. In addition, a Ce:YAG beam profile monitor and quadrant beam position monitors (QBPMs) were also installed upstream and downstream of the $\mathrm{KB}$ mirrors to monitor the beam position and intensity loss during reflection by the KB mirrors. In the QBPM, more than $99 \%$ of the X-rays were transmitted through a thin silicon nitride membrane, and the backscattered $\mathrm{X}$-rays were measured using four photodiodes. The intensity loss caused by the KB mirrors was measured by the QBPMs to be about $5 \%$. Considering that in our experiments we used a bare area of quartz substrate with a reflectance drop of about $5 \%$, we were still able to confirm that most of the X-rays entered through the mirror aperture.

In order to precisely align the focusing mirrors, the mirror manipulator shown in Fig. 2 was developed along with the focusing mirrors. The mirror manipulator has five degrees of freedom ( $X, Y$, yaw, pitch and roll) for each VFM and HFM. The distance between the VFM and HFM, which is associated with the $Z$-translation, was fixed at $30 \mathrm{~mm}$ (Fig. 2). The motion precision of the mirror manipulators was measured based on the angle variation of the autocollimator and linear encoder. The linear encoder recognizes each motion inside the vacuum chamber, and the autocollimator measures the reflected value through the viewport outside the chamber. The motion test results of the KB mirror manipulator show that the resolution of the linear actuator for $X$ - and $Y$-translation were $21.44 \mathrm{~nm}$ and $10 \mathrm{~nm}$, respectively. The angular resolution of the pitch, roll and yaw were $22 \mathrm{nrad}, 86 \mathrm{nrad}$ and $30 \mathrm{nrad}$, respectively. As a result, all manipulator motions were suitable for use during KB mirror alignment.

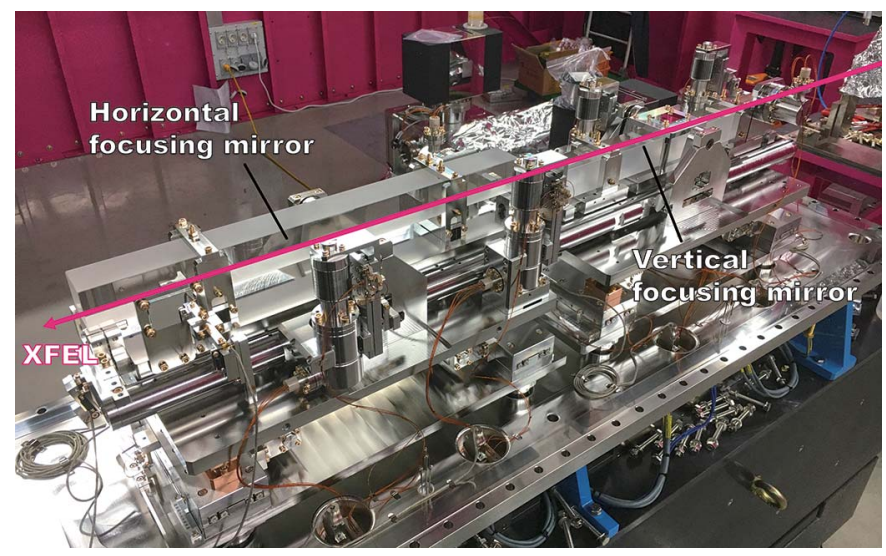

Figure 2

Photograph of the precision mirror manipulator with the elliptical VFM and HFMs at the NCI hutch at the PAL-XFEL.

In order to measure the focused beam size, a wire scanning method was used with a $200 \mu \mathrm{m}$-diameter tungsten wire that was placed on a high-resolution motion stage at the focal point. A PIN photodiode placed behind the wire was also used to measure the beam intensity while scanning the wire. In this measurement procedure, a double-side polished single-crystal silicon attenuator was used to avoid ablation of the wire by the focused beam. The measured intensity profiles of the focused beam are shown in Fig. 3. Each point represents the averaged intensity of ten pulses. The focused beam size was determined to be $1.94 \mu \mathrm{m}(\mathrm{H}) \times 2.08 \mu \mathrm{m}(\mathrm{V}) \mathrm{FWHM}$, which is in good agreement with the designed values.

\section{Summary}

The main optical components and the microfocusing $\mathrm{KB}$ mirror system installed at the NCI hutch at the PAL-XFEL are described. The XFEL was focused at the target beam size $(2 \mu \mathrm{m})$ through the developed mirror manipulator. In addition, this paper describes the optical configuration (polychromatic or monochromatic X-rays) that can be used in the NCI experimental hutch, and the diagnostic devices located upstream and downstream of the KB mirror system. These results will be useful for studies that will be performed at the NCI hutch.

\section{Acknowledgements}

We would like to acknowledge all members of the PAL-XFEL. This work was carried out with the support of Pohang Accelerator Laboratory.

\section{Funding information}

Funding for this research was provided by: Ministry of Science, ICT and Future Planning (MSIP), the Republic of Korea, through the PAL-XFEL project. 

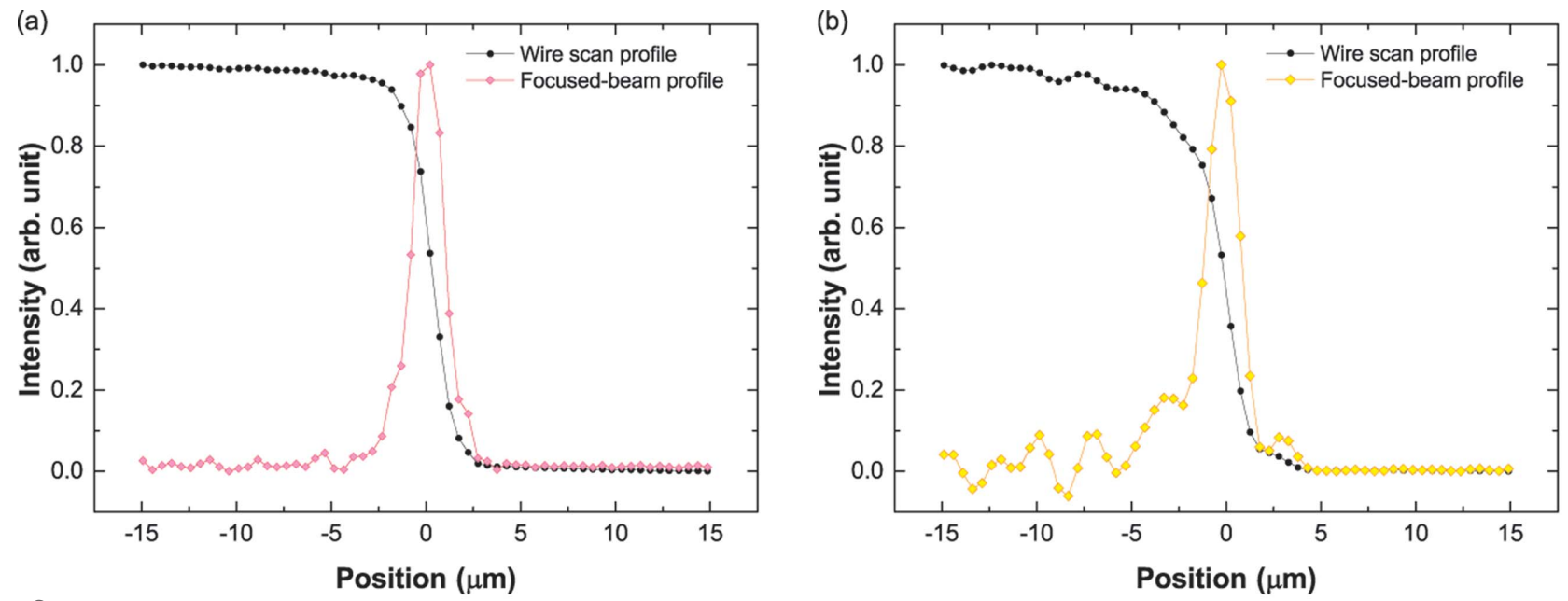

Figure 3

Typical focused-beam profiles measured horizontally $(a)$ and vertically $(b)$ with the wire scanning method. The measured beam size was $1.94 \mu \mathrm{m}$ $(\mathrm{FWHM})$ in the horizontal direction $(a)$, and $2.08 \mu \mathrm{m}(\mathrm{FWHM})$ in the vertical direction $(b)$.

\section{References}

David, C., Gorelick, S., Rutishauser, S., Krzywinski, J., VilaComamala, J., Guzenko, V. A., Bunk, O., Färm, E., Ritala, M., Cammarata, M., Fritz, D. M., Barrett, R., Samoylova, L., Grünert, J. \& Sinn, H. (2011). Sci. Rep. 1, 57.

Decking, W. \& Limberg, T. (2013). Report XFEL EU TN-2013-00401. European X-ray Free-Electron Laser Facility, Hamburg, Germany.

Emma, P. et al. (2010). Nat. Photon. 4, 641-647.

Ishikawa, T. et al. (2012). Nat. Photon. 6, 540-544.

Ko, I. S. et al. (2017). Appl. Sci. 7, 479.

McNeil, B. W. J. \& Thompson, N. R. (2010). Nat. Photon. 4, 814821.

Milne, C. J. et al. (2017). Appl. Sci. 7, 720.

Mimura, H., Yumoto, H., Matsuyama, S., Koyama, T., Tono, K., Inubushi, Y., Togashi, T., Sato, T., Kim, J., Fukui, R., Sano, Y.,
Yabashi, M., Ohashi, H., Ishikawa, T. \& Yamauchi, K. (2014). Nat. Commun. 5, 3539.

Neutze, R., Wouts, R., van der Spoel, D., Weckert, E. \& Hajdu, J. (2000). Nature (London), 406, 752-757.

Nilsson, D., Uhlén, F., Holmberg, A., Hertz, H. M., Schropp, A., Patommel, J., Hoppe, R., Seiboth, F., Meier, V., Schroer, C. G., Galtier, E., Nagler, B., Lee, H. J. \& Vogt, U. (2012). Opt. Lett. 37, 5046-5048.

Parc, Y. W., Han, J.-H., Kang, H. S., Kim, I., Yang, H., Hwang, I., Shim, C. H. \& Ko, I. S. (2014). J. Korean Phys. Soc. 64, 976-981.

Park, J., Eom, I. et al. (2016). Nucl. Instrum. Methods Phys. Res. A, 810, 74-79.

Park, J., Kim, S., Nam, K.-H., Kim, B. \& Ko, I. S. (2016). J. Korean Phys. Soc. 69, 1089-1093.

Reiche, S. (1999). Nucl. Instrum. Methods Phys. Res. A, 429, 243-248.

Schropp, A. et al. (2013). Sci. Rep. 3, 1633.

Yumoto, H. et al. (2013). Nat. Photon. 7, 43-47. 